\title{
Genetic architecture of rind penetrometer resistance in two maize recombinant inbred line populations
}

Kun $\mathrm{Li}^{1}$, Jianbing $\mathrm{Yan}^{2}$, Jiansheng $\mathrm{Li}^{\mathrm{i}^{*}}$ and Xiaohong Yang ${ }^{1^{*}}$

\begin{abstract}
Background: Maize (Zea Mays L.) is one of the most important cereal crops worldwide and provides food for billions of people. Stalk lodging can greatly undermine the standability of maize plants and therefore decrease crop yields. Rind penetrometer resistance is an effective and reliable method for evaluating maize stalk strength, which is highly correlated with stalk lodging resistance. In this study, two recombinant inbred line populations were constructed from crosses between the H127R and Chang7-2 lines, and between the B73 and By804 lines. We genotyped these two populations and their parents using 3,072 single nucleotide polymorphism markers and performed phenotypic assessment of rind penetrometer resistance in multiple environments to dissect the genetic architecture of rind penetrometer resistance in maize.

Results: Based on two linkage maps of 1,397.1 and 1,600.4 CM with average interval of 1.7 and $2.1 \mathrm{cM}$ between adjacent makers, respectively, seven quantitative trait loci (QTL) for rind penetrometer resistance were detected in the two recombinant inbred line populations. These QTL were distributed in seven genomic regions, and each accounted for $4.4-18.9 \%$ of the rind penetrometer resistance variation. The QTL with the largest effect on rind penetrometer resistance, aRPR3-1, was located on chromosome 3 with the flanking markers PZE-103123325 and SYN23245. This locus was further narrowed down to a 3.1-Mb interval by haplotype analysis using high-density markers in the target region. Within this interval, four genes associated with the biosynthesis of cell wall components were considered as potential candidate genes for the rind penetrometer resistance effect.

Conclusions: The inheritance of rind penetrometer resistance is rather complex. A few large-effect quantitative trait loci, together with a several minor-effect QTL, contributed to the phenotypic variation in rind penetrometer resistance in the two recombinant inbred line populations that were examined. A potential approach for improving stalk strength and crop yields in commercial maize lines may be to introgress favorable alleles of the locus that was found to have the largest effect on rind penetrometer resistance ( $q R P R 3-1$ ).
\end{abstract}

Keyword: Maize, Rind penetrometer resistance, QTL, SNP

\section{Background}

Plant lodging is a complicated phenomenon that is affected by several factors, including genetics, environment and field management. Lodging is a considerable challenge for main crops during the growth as it often causes severe reduction in yields. In maize (Zea Mays L.), stalk lodging, breakage that occurs at or below the ear, can lead to loss of ears at harvest $[1,2]$. It is estimated that yield

\footnotetext{
*Correspondence: lijiansheng@cau.edu.cn; yxiaohong@cau.edu.cn ${ }^{1}$ National Maize Improvement Center of China, Beijing Key Laboratory of Crop Genetic Improvement, China Agricultural University, Beijing 100193, China Full list of author information is available at the end of the article
}

losses caused by stalk lodging range from 5 to $20 \%$ worldwide $[1,3]$. Additionally, stalk lodging poses an obstacle to mechanized harvesting, and consequently increases labor costs. Thus, improving stalk-lodging resistance has become a key target for maize breeding programs.

Developing an effective and accurate way to evaluate stalk-lodging resistance is a critical issue in improving maize stalk strength. Numerous quantitative methods have been developed to predict stalk lodging resistance potential, which mainly include chemical methods based on analysis of stalk chemical composition and anatomical structures, and mechanical methods based 
on measurements of stalk breaking, bending, penetration and crushing [4-9]. Among the mechanical methods, rind thickness and crushing strength have been useful in increasing lodging resistance in maize as they have shown a strong relationship with stalk lodging $[7,9]$. However, these two methods are not ideal because they require the destruction of maize stalk. More recently, an efficient and non-destructive measure, rind penetrometer resistance (RPR), was developed to assess stalk strength [10]. Increased RPR shows a high correlation with stalk-lodging resistance [11-15] and this method has been widely applied in estimating stalk lodging resistance potential in maize [2,16-18] and in breeding maize hybrids that are highly resistant to stalk lodging $[1,11,12,19,20]$.

Despite these advances in measuring stalk lodging, little was known about the genetic basis of stalk lodging and RPR variation until quantitative trait loci (QTL) mapping was applied to RPR. The first of these studies identified 35 individual QTL and 11 pairs of epistatic interactions associated with RPR in four $\mathrm{F}_{2: 3}$ populations derived from B73, Mo47 and four inbred maize lines selected for stalk strength diversity [2]. The majority of these QTL explained $<15 \%$ of the phenotypic variation in RPR. An additional nine individual QTL and one more pair of epistatic interactions were detected in a recombinant inbred line (RIL) population crossed with a high-oil inbred line and B73, which account for another 1.15$12.43 \%$ of the phenotypic variation [17]. Recently, 18 QTL and 141 significant associations for RPR were identified using a nested association mapping panel containing 4,536 lines and 174 intermated B73 $\times$ Mo17 RILs. Only 10 QTL were shared between two populations or two studies, reflecting the complex nature of stalk lodging $[2,17,18]$.

The availability of the complete maize genome sequence [21] and haplotype maps [22,23] have facilitated the development of single nucleotide polymorphism (SNP) genotyping technologies for maize [24]. SNPs have become widely used markers in investigations of genetic variation and in linkage and association analyses in maize [25-32]. Compared with simple sequence repeat markers, SNPs are more accurate, less time-consuming and lower cost to identify, and better suited to high throughput genotyping platforms [33-35]. Currently, two GoldenGate assays containing 1,536 SNPs each and one Infinium BeadChip containing 56,110 SNPs have been developed for maize $[34,36,37]$. These SNP assays have been successfully used to examine the population structure and estimate genetic diversity of maize populations [34,36,37], and to identify variants associated with maize agronomic and quality traits [25-32].

In this study, we developed two RIL populations, H127R $\times$ Chang7-2 (referred to as POP-HRC) and B73 $\times$ By804 (referred to as POP-BYB), from four inbred lines with varying stalk strengths, and genotyped them using a GoldenGate maize SNP assay containing 3,072 SNPs to increase QTL resolution. Our objectives were to (1) identify QTL associated with RPR of maize stalks; (2) dissect the main-effect QTL with detailed haplotype in the target region; and (3) mine candidate genes associated with maize stalk strength.

\section{Results}

\section{Phenotypic variation in RPR}

Significant difference in RPR was observed between the H127R and Chang7-2 parental lines, whereas no significant difference was observed between the B73 and By804 lines (Table 1). Among these four parental lines, H127R, which is highly resistant to stalk lodging, had the highest RPR $\left(37.64 \pm 6.18 \mathrm{~N} / \mathrm{mm}^{2}\right)$, followed by Chang7-2 $\left(23.25 \pm 2.21 \mathrm{~N} / \mathrm{mm}^{2}\right)$, By804 $\left(21.67 \pm 2.63 \mathrm{~N} / \mathrm{mm}^{2}\right)$ and B73 $\left(21.09 \pm 2.82 \mathrm{~N} / \mathrm{mm}^{2}\right)$. The mean RPR value for the H127R $\times$ Chang7-2 RIL population (hereafter referred to as POP-HRC) was lower than the mean parent value, and the mean RPR value for the B73 $\times$ By804 RIL population (hereafter referred to as POP-BYB) was higher than the mean parent value (Table 1). RPR in both RIL populations showed a wide range with a normal distribution (Figure 1). Highly significant effects of genotype, environment, and genotype $\times$ environment interactions on RPR were observed in POP-HRC. In POP-BYB, both genotype and environment showed significant effects on RPR (Table 1). Broad-sense heritability estimates were $81.5 \%$ and $74.6 \%$ for POP-HRC and POP-BYB, respectively (Table 1).

\section{Summary of SNPs and genetic linkage maps}

A total of 2,866 and 3,029 high-quality SNPs were detected for POP-HRC and POP-BYB, respectively. The missing rate for these SNPs ranged from 0 to $15.35 \%$ in POP-HRC (average $0.83 \%$ ) and from 0 to $17.02 \%$ in POP-BYB (average 0.90\%). The minor allele frequency (MAF) for these SNPs ranged from 0 to 0.5 in both populations, with an average of 0.21 in POP-HRC and 0.23 in $\mathrm{POP}-\mathrm{BYB}$ and the heterozygosity ranged from 0 to $13.49 \%$ in POP-HRC (average 2.38\%) and from 0 to $4.79 \%$ in POP-BYB (average 0.92\%) (Table 2). 6\% (12/ 200) RILs of POP-BYB with SNP heterozygosity $\geq 0.1$ were excluded for further analysis. Of the high-quality SNPs, 2,252 SNPs were polymorphic in one or both of the RIL populations. 1,391 SNPs (45.3\%) were polymorphic between the parents of POP-HRC, 1578 SNPs (51.4\%) were polymorphic between the parents of POPBYB, and 717 SNPs (23.3\%) were polymorphic in both RIL populations.

After quality control, 822 SNPs in POP-HRC (215 lines), and 756 SNPs in POP-BYB (188 lines) were used to construct linkage maps. The total length of the linkage map for POP-HRC was 1,397.1 cM with an average 
Table 1 Descriptive statistics and broad-sense heritability for RPR in two RIL populations

\begin{tabular}{|c|c|c|c|c|}
\hline Population & POP-HRC & & POP-BYB & \\
\hline \multicolumn{5}{|l|}{ Parents } \\
\hline \multirow[t]{2}{*}{ Means $\pm \mathrm{SD}\left(\mathrm{N} / \mathrm{mm}^{2}\right)$} & H127R & $37.64 \pm 6.18$ & By804 & $21.67 \pm 2.63$ \\
\hline & Chang7-2 & $23.25 \pm 2.21$ & B73 & $21.09 \pm 2.82$ \\
\hline \multicolumn{5}{|l|}{ RILs } \\
\hline Population mean $\pm \mathrm{SD}\left(\mathrm{N} / \mathrm{mm}^{2}\right)$ & $27.19 \pm 2.12$ & & $22.95 \pm 1.03$ & \\
\hline Range $\left(\mathrm{N} / \mathrm{mm}^{2}\right)$ & $22.59-33.65$ & & $20.75-26.13$ & \\
\hline F value Environment & $397.20^{* *}$ & & $116.43^{* *}$ & \\
\hline Genotype & $9.51^{* *}$ & & $3.94^{* *}$ & \\
\hline Environment $\times$ Genotype & $1.76^{* *}$ & & & \\
\hline Replication (environment) & 3.75 & & & \\
\hline Heritability $\left(H^{2}\right)^{\text {a }}(\%)$ & 81.5 & & 74.6 & \\
\hline Confidence interval $^{b}$ & $77.5-84.8$ & & $69.2-78.8$ & \\
\hline
\end{tabular}

**Significant at $\mathrm{P}<0.01$.

a Broad-sense heritability $\left(H^{2}\right)$ of RPR.

${ }^{b} 90 \%$ confidence intervals of broad-sense heritability.

interval of $1.7 \mathrm{cM}$, and the total length of the map for POP-BYB was 1,600.4 cM with an average interval of $2.1 \mathrm{cM}$ (Table 3, Additional file 1). The relative locations of $86.3 \%$ (710) of the SNPs in POP-HRC and $68.1 \%$ (515) of the SNPs in POP-BYB were the same as their physical locations in the B73 reference genome Version 5 b.60 [38]. Among these mapped SNPs, 37.0\% (304) in POP-HRC and $24.9 \%$ (188) in POP-BYB showed segregation distortion at $\mathrm{P}<0.05$, which formed nine and six hot blocks of segregation distortion, respectively (Additional file 1).

\section{QTL analysis}

In total, seven QTL were detected that appeared to be associated with RPR in the two RIL populations (Table 4, Additional file 1). The empirical threshold logarithm of odds (LOD) values for the genome-wide significance $(\mathrm{P}<0.05)$ were determined to be 3.1 for POP-HRC and 3.2 for POP-BYB after 1000 permutations. These seven
QTL were distributed in seven genomic regions across five chromosomes with marker intervals ranging from 0.6 to 24.9 Mb. The phenotypic variation in RPR explained by each QTL ranged from 4.4\% (qPPR2) to $18.9 \%$ ( $q P P R 3-1$ ). We identified three main-effect QTL in three chromosomal regions which accounted for $>10 \%$ of the RPR variation.

In POP-HRC, four of the RPR-associated QTL, located on chromosomes 2, 3 and 9 (Table 4, Additional file 1), together explained $50.4 \%$ of the phenotypic variation. The QTL on chromosome 3 flanked by the PZE103123325 and SYN23245 markers, qRPR3-1, had the largest effect and accounted for $18.9 \%$ of the phenotypic variation in RPR. The H127R allele at this locus was correlated with a $1.05 \mathrm{~N} / \mathrm{mm}^{2}$ increase in RPR. The second largest-effect QTL for RPR, qRPR9, which explained $8.1 \%$ of the phenotypic variation, was located between PZE-109058177 and PZE-109076761 on chromosome 9.

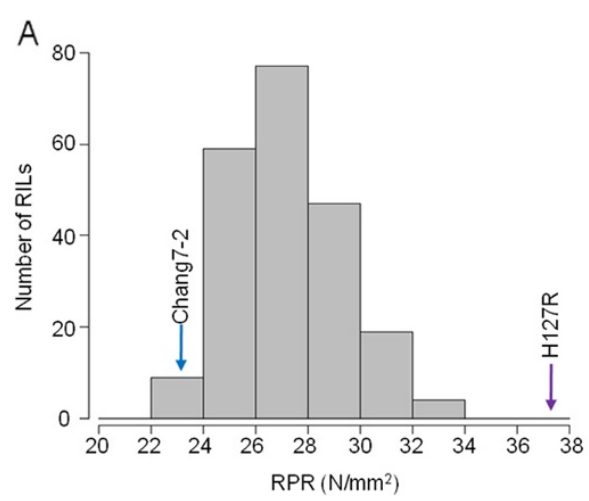

B

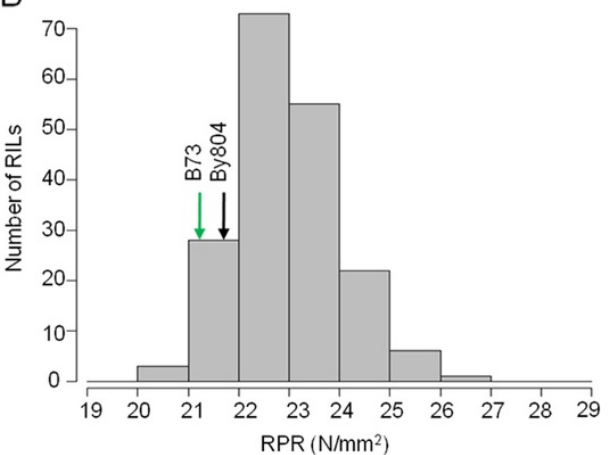

Figure 1 Frequency distribution of RPR for RILs in the two populations in all environments. (A) POP-HRC. (B) POP-BYB. Parental strain values are indicated with arrows. 
Table 2 Summary of SNP characteristics in the two RIL populations

\begin{tabular}{|c|c|c|c|c|}
\hline & & & & \\
\hline & Mean \pm SD & Range & Mean \pm SD & Range \\
\hline High-quality SNP number & 2866 & & 3029 & \\
\hline SNP missing rate (\%) & $0.83 \pm 1.44$ & $0.00-15.35$ & $0.90 \pm 1.60$ & $0.00-17.02$ \\
\hline MAF of SNPS & $0.21 \pm 0.22$ & $0.00-0.50$ & $0.23 \pm 0.22$ & $0.00-0.50$ \\
\hline SNP heterozygosity (\%) & $2.38 \pm 2.86$ & $0.00-13.49$ & $0.92 \pm 1.10$ & $0.00-4.79$ \\
\hline SNP missing rate in each line (\%) & $0.83 \pm 0.53$ & $0.07-3.49$ & $0.90 \pm 2.46$ & $0.00-17.70$ \\
\hline SNP heterozygosity in each line (\%) & $2.40 \pm 1.48$ & $0.10-7.68$ & $0.92 \pm 1.12$ & $0.00-5.88$ \\
\hline
\end{tabular}

The remaining two QTL, $q R P R 2$ on chromosome 2 and qPPR3-2 on chromosome 3, explained $4.4 \%$ and $6.7 \%$ of the phenotypic variation, respectively. The alleles that were associated with increased RPR at these two loci also came from H127R.

In POP-BYB, the remaining three RPR-associated QTL, qRPR4, qRPR6-1 and qRPR6-2, accounted for $31.7 \%$ of the phenotypic variation. The $\mathrm{B} 73$ alleles at $q R P R 4$ and qRPR6-2 were correlated with similar $0.39 \mathrm{~N} / \mathrm{mm}^{2}$ increases in RPR. The By804 allele at qRPR6-1, had an additive effect of $0.27 \mathrm{~N} / \mathrm{mm}^{2}$ for increased RPR.

To further confirm the seven RPR-associated QTL identified using the best linear unbiased prediction (BLUP) values, we also mapped RPR-associated QTL in the RIL populations grown in different environments and replications grown in the same environments (Additional file 2). The association with RPR was stable for $q R P R 3-1$ in all environments/replications, whereas the remaining six QTL differed significantly in at least two environments/replications or showed obvious LOD peaks in different environments/ replications. In addition to the original seven QTL, 13 QTL were identified that associated with RPR in one or two environments/replications.
Beyond individual QTL, one pair of epistatic QTL between $q R P R 3-1$ and $q R P R 3-2$ was detected in POP-HRC. The type of epistasis between $q R P R 3-1$ and $q R P R 3-2$ was additive interacted by additive. This pair of epistatic QTL explains $2.5 \%$ of the phenotypic variation with a positive effect on RPR coming from the parental digenic combination. None epistatic QTL were identified in POP-BYB.

\section{Fine mapping of $q R P R 3-1$ in POP-HRC}

Because of the large effect of $q R P R 3-1$ and the high density of SNP markers available at this locus, we were able to precisely determine the critical recombination breakpoint using SNPs that were polymorphic between two parents of POP-HRC in the QTL interval. Initially, qRPR3-1 was localized to between the SNP makers PZE103104806 (M1) and PZE-103132112 (M10), with the LOD values of all SNP markers in this interval greater than 3.1 (Figure 2A). This region spanned a genetic distance of $27.9 \mathrm{cM}$, corresponding to a physical distance of $21.9 \mathrm{Mb}$ in the B73 reference sequence Version $5 \mathrm{~b} .60$ [38]. Using 10 polymorphic SNP markers in this region, 20 haplotypes were observed for the 215 RILs in POP-HRC. Of these haplotypes, only 12 had only one recombination

Table 3 Summary of the linkage map characteristics of the two RIL populations

\begin{tabular}{|c|c|c|c|c|c|c|c|c|c|c|}
\hline \multirow[b]{2}{*}{ Chromosome } & \multicolumn{5}{|c|}{ POP-HRC } & \multicolumn{5}{|c|}{ POP-BYB } \\
\hline & $\begin{array}{c}\text { Number } \\
\text { of markers }\end{array}$ & $\begin{array}{l}\text { Length } \\
\text { (cM) }\end{array}$ & $\begin{array}{c}\text { Average } \\
\text { interval (cM) }\end{array}$ & $\begin{array}{c}\text { Minimum } \\
\text { interval (cM) }\end{array}$ & $\begin{array}{c}\text { Maximum } \\
\text { interval (cM) }\end{array}$ & $\begin{array}{l}\text { Number } \\
\text { of markers }\end{array}$ & $\begin{array}{l}\text { Length } \\
\text { (cM) }\end{array}$ & $\begin{array}{c}\text { Average } \\
\text { interval (cM) }\end{array}$ & $\begin{array}{c}\text { Minimum } \\
\text { interval (cM) }\end{array}$ & $\begin{array}{c}\text { Maximum } \\
\text { interval (cM) }\end{array}$ \\
\hline 1 & 99 & 182.4 & 1.8 & 0.2 & 7.5 & 117 & 253.1 & 2.2 & 0.3 & 13.3 \\
\hline 2 & 96 & 162.2 & 1.7 & 0.2 & 14.0 & 74 & 178.5 & 2.4 & 0.3 & 15.4 \\
\hline 3 & 80 & 171.6 & 2.2 & 0.2 & 9.7 & 60 & 184.4 & 3.1 & 0.3 & 14.3 \\
\hline 4 & 88 & 155.8 & 1.8 & 0.2 & 11.5 & 84 & 141.0 & 1.7 & 0.3 & 8.2 \\
\hline 5 & 95 & 168.0 & 1.8 & 0.3 & 8.7 & 91 & 171.2 & 1.9 & 0.3 & 14.6 \\
\hline 6 & 75 & 117.9 & 1.6 & 0.2 & 8.3 & 83 & 148.2 & 1.8 & 0.3 & 15.0 \\
\hline 7 & 73 & 131.8 & 1.8 & 0.2 & 11.8 & 90 & 172.5 & 1.9 & 0.3 & 12.0 \\
\hline 8 & 87 & 111.7 & 1.3 & 0.2 & 13.2 & 73 & 140.5 & 1.9 & 0.3 & 11.9 \\
\hline 9 & 81 & 111.4 & 1.4 & 0.1 & 13.1 & 50 & 125.7 & 2.5 & 0.3 & 11.4 \\
\hline 10 & 48 & 84.3 & 1.8 & 0.2 & 12.3 & 34 & 85.3 & 2.5 & 0.3 & 14.2 \\
\hline All & 822 & 1,397.1 & 1.7 & & & 756 & $1,600.4$ & 2.1 & & \\
\hline
\end{tabular}


Table 4 RPR-associated QTL in the two RIL populations

\begin{tabular}{|c|c|c|c|c|c|c|c|c|c|}
\hline Population & QTL & Chromosome & Peak $^{a}(\mathrm{cM})$ & Marker interval & $\begin{array}{l}\text { Genetic } \\
\text { interval (cM) }\end{array}$ & $\begin{array}{l}\text { Physical } \\
\text { position }^{\mathrm{b}}(\mathrm{Mb})\end{array}$ & LOD & $A^{c}$ & $R^{2}(\%)^{d}$ \\
\hline \multirow[t]{5}{*}{ POP-HRC } & $q R P R 2$ & 2 & 162.1 & SYN6917-PZE102193611 & $160.1-162.2$ & $236.4-237.0$ & 3.8 & 0.45 & 4.4 \\
\hline & qRPR3-1 & 3 & 107.4 & PZE-103123325-SYN23245 & $104.5-111.1$ & $181.1-184.7$ & 14.0 & 1.05 & 18.9 \\
\hline & qRPR3-2 & 3 & 133.9 & PZE-103156977-PZE-103160158 & $132.4-134.2$ & $209.1-211.2$ & 5.9 & 0.61 & 6.7 \\
\hline & qRPR9 & 9 & 47.0 & PZE-109058177-PZE-109076761 & $42.4-50.0$ & $99.4-124.3$ & 6.6 & 0.66 & 8.1 \\
\hline & Total $^{e}$ & & & & & & & & 50.4 \\
\hline \multirow[t]{4}{*}{ POP-BYB } & qRPR4 & 4 & 55.7 & PZE-104080388-PZE-104084757 & $50.3-55.7$ & $154.7-158.7$ & 7.9 & -0.39 & 14.0 \\
\hline & qRPR6-1 & 6 & 89.4 & PZE-106088503-SYN4646 & $88.5-91.9$ & $146.1-147.7$ & 3.6 & 0.27 & 6.0 \\
\hline & qRPR6-2 & 6 & 143.3 & SYN34377-PHM3466.69 & $133.3-148.2$ & $163.2-167.0$ & 6.2 & -0.39 & 13.8 \\
\hline & Total $^{e}$ & & & & & & & & 31.7 \\
\hline
\end{tabular}

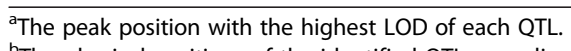

${ }^{\mathrm{b}}$ The physical positions of the identified QTL according to B73 reference sequence Version 5.60 [38].

c Additive effect of the identified QTL: a positive value indicates that the alleles from H127R and By804 increases RPR, and a negative value indicates that the alleles from Chang7-2 and B73 increase RPR.

${ }^{d}$ Percentage of phenotypic variation explained by additive effects of the identified QTL.

'Total percentage of phenotypic variation explained by all QTL computed by MIM.

breakpoint in the QTL interval (Figure 2B). We next compared the mean RPR, estimated using the BLUP values, of individuals with and without H127R alleles using a twosample $t$-test. We observed that the RPR values of haplotypes $4-8\left(28.28-30.60 \mathrm{~N} / \mathrm{mm}^{2}\right)$ were significantly higher than the RPR value of haplotype $1\left(26.24 \mathrm{~N} / \mathrm{mm}^{2}\right)$, which did not carry H127R alleles at any of the 10 SNPs $(\alpha=0.05$, $\left.\mathrm{P}=1.13 \times 10^{-2}-4.32 \times 10^{-8}\right)$, whereas haplotypes 2 , 3 , and 9-12 showed similar RPR values $\left(25.14-27.41 \mathrm{~N} / \mathrm{mm}^{2}\right)$ to haplotype 1 . Therefore, we were able to narrow the location of $q R P R 3-1$ to a 3.1-Mb window between the markers PZE103123992 (M8) and SYN23245 (M9). To further confirm the interval narrowed down, we also performed haplotype analysis using the RPR value in each environment, and the identity interval was inferred (data unpublished).

\section{Candidate genes in the target QTL region}

Based on the available annotation of the B73 reference sequence Version 5b.60 [38], there are 86 predicted genes in the 3.1-Mb target region (Additional file 3). Of these genes, 32 encode proteins of unknown function and the remaining 54 encode proteins that could be classified into four categories (Figure 3); protein kinases, enzymes involved in cell wall component synthesis and degradation, transcription factors, and enzymes related to other biological pathways.

\section{Discussion}

\section{Genetic characterization of RPR in maize}

Precise phenotypic measures are crucial for genotypephenotype association analysis [39]. Previous studies have shown that RPR is highly associated with stalk-lodging resistance in maize $[8,11,12,20]$. For example, divergent selection for stalk crushing strength in synthetic maize populations has resulted in increased RPR [6,40]. In addition, RPR of the internodes below the uppermost ear has been found to be highly correlated with the RPR of internodes between the last ear and ground on maize plants [41]. Thus, measuring RPR of the internode below the uppermost ear is suggested to be one of the best ways to evaluate stalk-lodging resistance in maize in the current status.

Although RPR is a complex quantitative trait that can be affected by environment, most of the phenotypic variation appears to be due to genetic factors. The broadsense heritability of RPR in maize, estimated in two previous studies as well as our study, reached over $90 \%$ in some segregating populations $[2,17]$. The high broad-sense heritability reflects the accuracy and feasibility of the method used to quantify RPR in these studies. Whereas, broadsense heritability values of RPR in maize estimated from nested association map families are far lower than the values we estimated, ranging from 8 to 34\% (averaged 21\%) across 26 RIL populations [18]. The reduced heritability values may be attributable to the different populations surveyed, differences in the growing environments, or to the relatively low number of replications examined for each line in their study [18]. Further characterization of RPR in more bi-parent segregating populations is needed to reconcile these differences in heritability values.

\section{The complex nature of RPR in maize}

The present study identified seven RPR-associated QTL were identified in two RIL populations. Among these QTL, only the largest-effect QTL, qRPR3-1, was also identified in two previous studies by Flint-Garcia et al. [2] and $\mathrm{Hu}$ et al. [17]; qRPR3-2 and $q R P R 6-2$ were also detected in the Flint-Garcia et al. study and were found to explain $6.7 \%$ and $13.8 \%$ of the phenotypic variation, respectively [2]. Our study revealed that a few large-effect QTL, together with some minor-effect QTL, provide most 


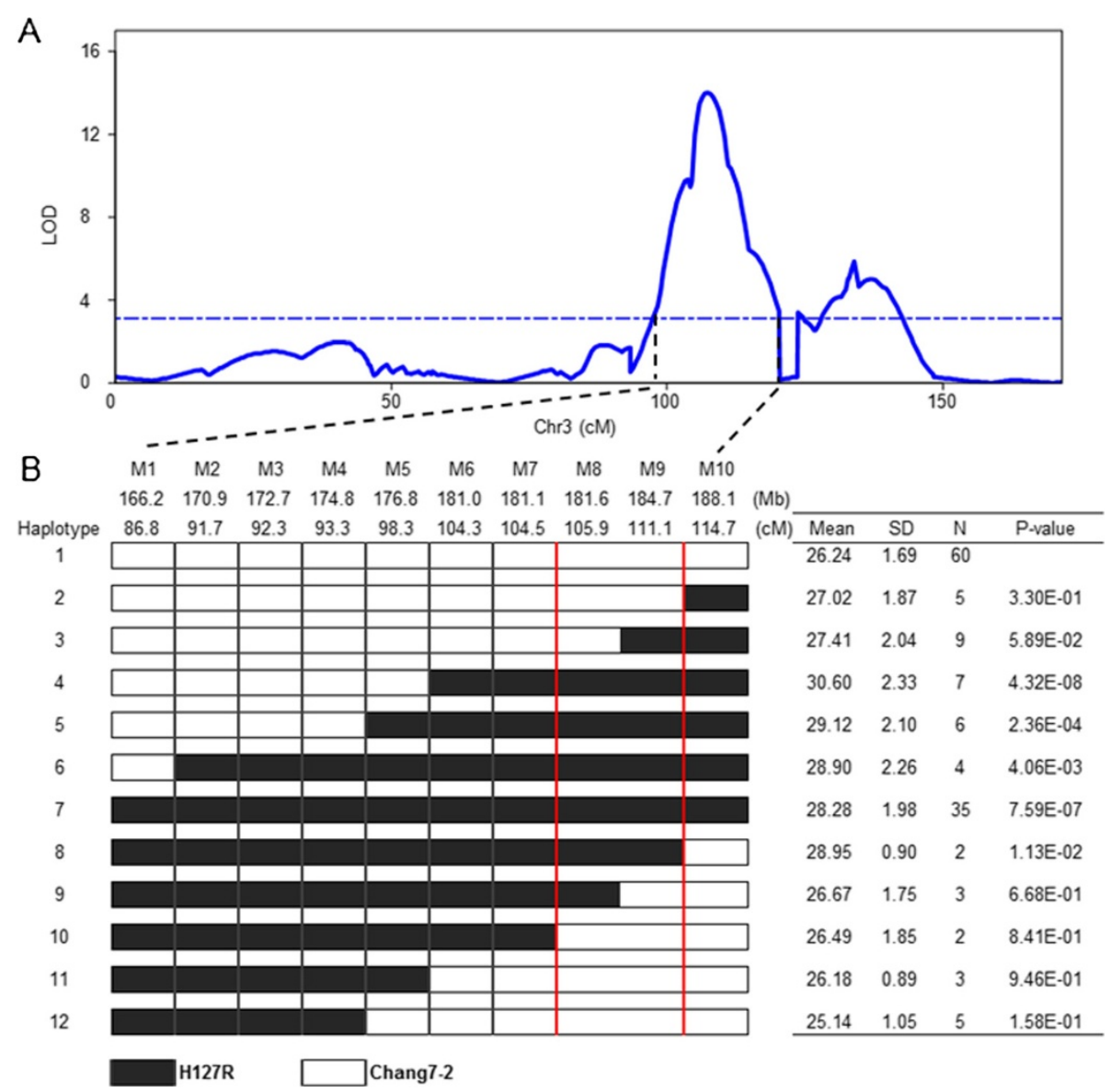

Figure 2 Haplotype analysis and fine mapping of qRPR3-1 in POP-HRC. (A) LOD profile for qRPR3-1 estimated using the BLUP values of plants grown in the three locations/years. (B) Detailed haplotype analysis of the putative RPR-associated interval with the BLUP value of RPR. The red lines indicate the narrowed interval of qRPR3-1, M1-M10 represent the SNP markers PZE-103104806, PZE-103110761, PZE-103112971, PZE-103114860, PZE-103118170, SYN31220, PZE-103123325, PZE-103123992, SYN23245 and PZE-103132112, respectively.

of the genetic basis of RPR, consistent with previous studies $[2,17,18]$. Together with this study, a total of 69 RPR-associated QTL have been identified in 33 segregating populations. The phenotypic variation explained by the largest-effect QTL in each population ranged from 5.6 to $20.2 \%$. Among these QTL, only $\sim 10$ were common in at least two populations. The low repeatability across populations may be due to the complex nature of RPR in maize, and the fact that most individual loci have small effects, which results in relatively small differences in RPR between parent strains $[2,17,18]$.

In addition to single-effect QTL for RPR, FlintGarcia et al. [2] and $\mathrm{Hu}$ et al. [17] detected 11 pairs of epistatic QTL in three $F_{2: 3}$ populations and one pair of epistatic QTL in one RIL population. The majority of these pairs of epistatic QTL explained $<10 \%$ of phenotypic variations in each population. These findings, together with one pair of epistatic QTL identified in our study, suggest that epistasis also contributes to the genetic basis of RPR, but the effect of epistasis is relatively weak and not consistent in different biparent populations.

\section{Pleiotropic loci for stalk components}

RPR is a physical measure of maize stalk strength, and significantly correlates with levels of some cell wall components $[17,42]$. Specifically, selection for stalk strength in a maize synthetic line using RPR found that divergent selection for increased RPR leads to increases in crude fiber, cellulose and lignin content in the plants [42]. Similarly, RPR significantly correlates with the levels of cell wall components, including acid detergent fiber, acid detergent lignin, crude fiber, cellulose, and neutral detergent fiber in internodes of a Ce03005/B73 RIL population [17]. The high correlation indicates that RPR-associated QTL may overlap, at least to some degree with QTL related to cell wall components of maize stalks.

Because RPR results from the combination of different cell wall component levels, it is likely that many of the same polymorphisms underlie the QTL associated with 


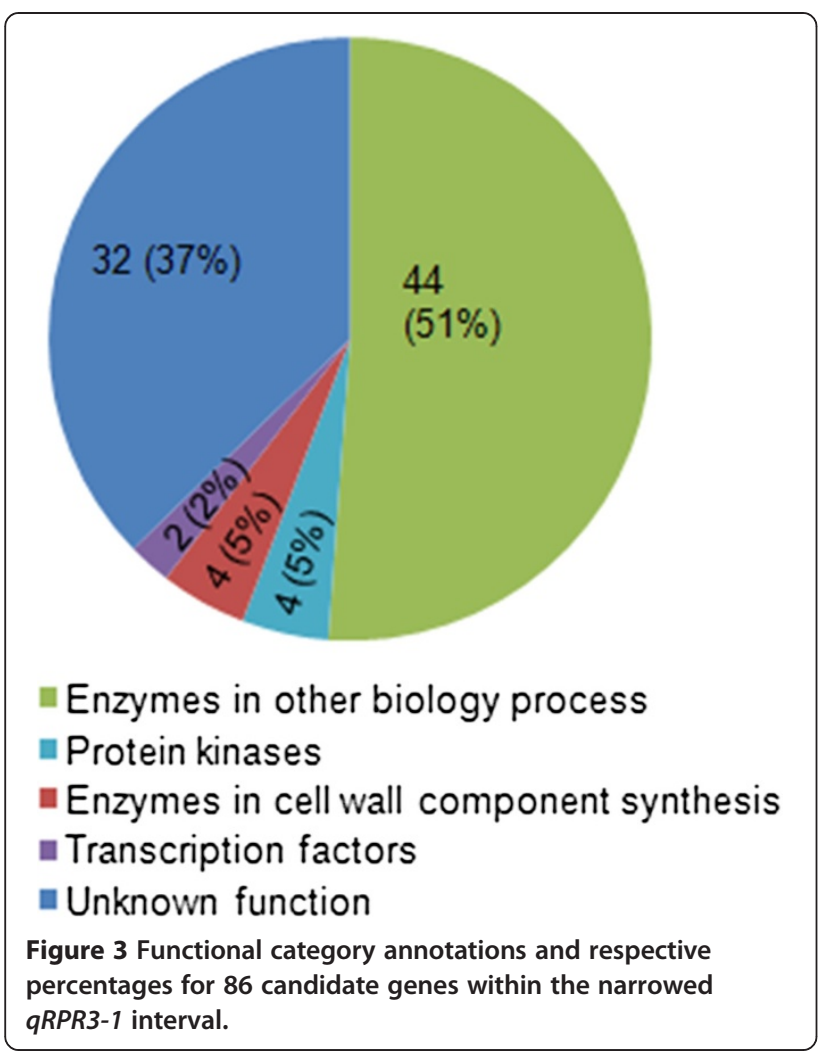

these two traits. Previous studies have identified 55 genomic regions related to cell wall components [43-45], including three loci in chromosome bins 3.06, 3.08 and 6.07 that are located near RPR-related genomic regions detected in our study. The largest-effect QTL for RPR in chromosome bin 3.06, qRPR3-1, co-localized with QTL clusters for acid detergent lignin and hemicelluloses detected in a F288 $\times$ F271 RIL $\times$ F286 top-cross population [45]. Another RPR-associated QTL in chromosome bin 3.08 in POP-HRC, $q R P R 3-2$, is close to a QTL for Klason lignin in an RIL population derived from a cross between an old Minnesota13 line and a modern Iodent line [43]. This QTL also co-localizes with the hemicelluloseassociated QTL in the F288 $\times$ F271 RIL $\times$ F286 top-cross population [45]. The POP-BYB QTL in chromosome bin 6.07, qRPR6-2, co-localized with a QTL that controls acid detergent fiber in De811 $\times$ B73 RIL populations [44]. Taken together, this information will help us to mine the candidate genes underlying QTL for RPR based on pleiotropy.

\section{Co-localization of RPR-related QTL and candidate genes}

Generally, the final goal of primary QTL mapping is to clone genes of interest. Association analysis combined with traditional linkage analysis can speed up the process of cloning genes [46]. Recently, Xu et al. [32] reported that haplotype analysis using high-density markers within the target QTL interval in segregation populations is an effective way to narrow down the primary QTL with large effects. Applying this strategy, we narrowed $q R P R 3-1$ to a $3.1-\mathrm{Mb}$ window predicted to contain 86 genes. Although this still leaves many possible genes within the loci that could be responsible for the RPR association of qRPR3-1, knowledge of connection between RPR and cell wall components can guide the choice of candidate genes for further study and potential cloning. Consequently, the four candidate genes known to be involved in cell wall component metabolism are considered the most likely to be the RPR-related qRPR3-1 gene. GRMZM2G132169 encodes a laccase that has been shown to play a role in lignin polymerization in Arabidopsis thaliana $[47,48]$. GRMZM2G359234 encodes a UDP-glucuronic acid decarboxylase that produces UDP-xylose, a substrate for xylan biosynthesis [49], and decreased xylan content leads to reduced stem mechanical strength [50]. GRMZM2G440016 encodes pectin methylesterase, which catalyses the de-esterification of pectin, and is significantly associated with stem strength in Arabidopsis thaliana [51]. GRMZM2G126077 encodes the precursor of pectate lyase, and is involved in the pectin biodegradation pathway. Although some evidence from Arabidopsis supports the idea that GRMZM2G359234 and GRMZM2G440016 are the top candidates to be the RPR-associated genes, further investigation is necessary to confirm this connection, such as candidate-gene association mapping, fine mapping, and functional validation. Besides these four candidate genes, the other genes of unknown function or genes with function not linked to cell wall metabolism may also be the true variant for $q R P R 3-1$.

\section{Application of RPR-related QTL to the improvement of maize stalk strength}

Stalk strength is an important factor in breeding maize varieties to maintain grain yield. Phenotypic selection based on RPR has been successful in improving stalk strength in several maize synthetic populations [11,12,19,20,42,52,53]. Marker-assisted selection (MAS) is an alternative way to improve target traits [54-57], including disease resistance in maize [58]. Flint-Garcia et al. compared the efficiency of MAS and phenotypic selection for RPR, and found that MAS was more efficient [1]. Therefore, understanding the genetic architecture of stalk strength will enhance efforts to optimize stalk strength, and ultimately mitigate stalk lodging. The large-effect QTL for RPR, $q R P R 3-1$, is a potential QTL for improving stalk strength via MAS. Additionally, qRPR3-1 was narrowed to a relatively small QTL interval, which reduces the probability of linkage drag with deleterious alleles. The genetic effects of $q R P R 3-1$ associated with maize stalk cell wall components will provide additional phenotypic markers to guide the introgression of favorable alleles at the $q R P R 3-1$ locus. 


\section{Conclusions}

In this study, we mapped RPR-associated QTL in two RIL populations using medium SNP-density based linkage maps. We found that four QTL in POP-HRC and three in POP-BYB explained $50.4 \%$ and $31.7 \%$ of RPR variation, respectively. Only one of the seven QTL accounted for $>15 \%$ of the RPR variations. These findings indicate that a few large-effect QTL and additional minor-effect QTL contribute to the phenotypic variation in RPR in the two RIL populations, reflecting the complex nature of stalk strength. The largest-effect QTL in chromosome bin 3.06 in POP-HRC, qRPR3-1, was narrowed to a $3.1-\mathrm{Mb}$ interval by haplotype analysis using high-density markers in the target QTL interval. Within this interval, four genes associated with the biosynthesis of cell wall component were considered the most likely candidate genes for the qRPR3-1 locus. This information will be valuable for introgressing favourable alleles of qRPR3-1 into elite inbred lines to enhance stalk strength, and in turn mitigate stalk lodging.

\section{Methods}

\section{Genetic materials}

One maize $\mathrm{F}_{9}$ RIL population, consisting of 200 lines, was derived from a cross between the B73 and By804 lines. B73 is an elite inbred line derived from the Iowa Stiff Stalk Synthetic maize population. By804 is an inbred line developed from a Beijing high-oil population. Due to the high heterozygosity of 12 RILs in this population $(>10 \%)$, only the remaining 188 lines were selected for subsequent analysis. Another $\mathrm{F}_{6}$ RIL population, containing 215 lines, was constructed by crossing the inbred lines H127R and Chang7-2. H127R is a parental line of the elite hybrid Zhongnongda 4, and Chang7-2 is the male parent line of the hybrid Zhengdan985. H127R is more resistant to stalk lodging than Chang7-2. For simplicity, we refer to the $\mathrm{B} 73 \times \mathrm{By} 804$ RIL population as POP-BYB, and the H127R $\times$ Chang7-2 RIL population as POP-HRC.

\section{Field experiments and phenotyping}

All 415 RILs, together with the four parent lines, were planted in a randomized complete block design from 2011 to 2013. For POP-HRC, two replications were planted in each of three environments, including Beijing in 2012 and in 2013 and Henan in 2013. For POP-BYB, one replication was planted in each of six environments, including Hainan in 2011 and in 2012, and Beijing, Henan, Chongqing and Yunnan in 2012. Each line was grown in a single $2.5 \mathrm{~m}$ row, rows were $0.67 \mathrm{~m}$ apart, and planting density was 45,000 plants/ha. The RPR of six randomly selected plants in each row was evaluated in the middle of the flat side of the internodes below the primary ear with an electronic penetrometer (AWOS-
SL04, Aiwoshi Company, Hebei, China) at two weeks after flowering at the average level of each population, which roughly corresponded with the milk stage.

\section{Phenotypic data analysis}

The variance components of RPR were estimated using PROC GLM in SAS 9.2 (SAS Institute). The model for variance analysis for POP-HRC was: $y_{i j k}=\mu+e_{l}+r_{k(l)}+f_{i}+$ $(f e)_{i l}+\varepsilon_{l i k}$, where $\mu$ is the grand mean of RPR, $f_{i}$ is the genetic effect of the "i"th line, $e_{l}$ is environmental effect of the "l"th environment, $(f e)_{i l}$ is the interaction effect between genetic and environmental effects, $r_{k(l)}$ is effect of replications within environments, and $\varepsilon_{l i k}$ is the residual error. For POP-BYB, the interaction effect between environment and genotype was treated as residual error due to the fact that there were no replications within each environment. These variance components were used to calculate broad-sense heritability based on the population means [59]. The broad-sense heritability in POP-HRC was estimated as $h^{2}=\sigma_{g}^{2} /\left(\sigma_{g}^{2}+\sigma_{g e}^{2} / e+\sigma_{\varepsilon}^{2} / r e\right)$, where $\sigma_{g}^{2}$ is the genetic variance, $\sigma_{g e}^{2}$ is the interaction of genotype with environment, $\sigma_{\varepsilon}^{2}$ is the residual error, $e$ and $r$ represent the number of environments and replications in each environment. In POP-BYB, the broad-sense heritability was estimated as $h^{2}=\sigma_{g}^{2} /\left(\sigma_{g}^{2}+\sigma_{\varepsilon}^{2} / e\right)$, where $\sigma_{g}^{2}$ is the genetic variance, $\sigma_{\varepsilon}^{2}$ is the residual error, $e$ stands for the number of environments. Confidence interval of $h^{2}$ were calculated according the method described by Knapp et al. [60].

A mixed linear model was fitted to each RIL to obtain the BLUP for RPR: $y_{i}=\mu+f_{i}+e_{i}+\varepsilon_{i}$, where $y_{i}$ is the phenotypic value of individual $\mathrm{i}, \mu$ is the grand mean for all environments, $f_{i}$ is the genetic effect, $e_{i}$ is effect of different environments, and $\varepsilon_{i}$ is the random error. The grand mean was fitted as a fixed effect, and genotype and environment were considered random effects. The MIXED procedure in SAS9.2 (SAS Institute) was used to obtain the BLUP value.

\section{Genotyping and genetic map construction}

Genomic DNA was extracted from leaf tissue of the RILs and parent lines using the modified CTAB method [61] and used for genotyping with the MaizeSNP3K subset (3,072 SNPs) of the Illumina MaizeSNP50 BeadChip [37]. SNP genotyping was performed on the Illumina GoldenGate SNP genotyping platform [62] at the National Maize Improvement Center of China, China Agricultural University. The quality of each SNP was checked manually as described by Yan et al. [34], and SNPs with poor quality were excluded for further analysis.

In each RIL population, the missing rate, MAF and heterozygosity for each SNP and the missing rate and 
heterozygosity for each line were calculated using PLINK packages [63]. The SNPs with missing rates $\leq 20 \%$ and MAFs $\geq 0.05$ were used to construct the genetic linkage map with JoinMap 4.0 [64], using the Kosambi mapping function for calculating map distances. Linkage groups were formed at a minimum LOD of 6 , and a regressionmapping algorithm was used to calculate map distances.

\section{QTL mapping}

Windows QTL Cartographer 2.5 [65] was used for QTL detection with the RPR BLUP values across the different populations, environments and replications. The whole genome scan was performed using composite interval mapping with a $0.5 \mathrm{cM}$ scanning interval between markers, and the window size was set at $10 \mathrm{cM}$. Model 6 of the Zmapqtl module was selected for detecting QTL and estimating their effects. Forward-backward stepwise regression with five controlling markers was used to control for background from flanking makers. After 1,000 permutations, the threshold LOD value was determined at a significance level of $\mathrm{P}<0.05$. The confidence interval of QTL position was determined with one-LOD support interval method [66]. To estimate the interactions of significant QTL and their total phenotypic variation, multiple interval mapping (MIM) in Windows QTL Cartographer 2.5 was performed with Bayesian Information Criteria (BIC-M0) as criteria of MIM model [67].

\section{Annotation of candidate genes}

Based on the information available in the MaizeSequence database [38], the function of each gene within the largest-effect QTL interval was inferred from orthologues in Arabidopsis or rice. Additional protein prediction information was obtained from the InterPro module in the European Bioinformatics Institute database (http:// www.ebi.ac.uk/interpro/) [68].

\section{Additional files}

\section{Additional file 1: Genetic maps and distribution of putative} RPR-related QTL in two RIL populations. (A) POP-HRC. (B) POP-BYB. The red bar on each chromosome indicates the hot block of segregation distortion, and the black bar indicates the location of the identified QTL, the blue oval represents the centromere of each chromosome

Additional file 2: LOD profiles of the identified RPR-associated QTL in the RIL populations grown in different environments. (A) POP-HRC. E1, 2013 Beijing replication 1; E2, 2013 Beijing replication 2; E3, 2013 Henan replication 1; E4, 2013 Henan replication 2; E5, 2012 Beijing replication 1; E6, 2012 Beijing replication 2; E7, BLUP. (B) POP-BYB. R1, 2011 Hainan; R2, 2012 Chongqing; R3, 2012 Yunnan; R4, 2012 Henan; R5, 2012 Beijing; R6, 2012 Hainan; R7, BLUP.

Additional file 3: Annotation of the 86 predicted genes located within the narrowed $q R P R 3-1$ interval in POP-HRC.

\section{Abbreviations}

RPR: Rind penetrometer resistance; QTL: Quantitative trait loci;

RIL: Recombinant inbred line; SNP: Single nucleotide polymorphism;
CM: centimorgan; MAF: Minor allele frequency; POP-HRC: H127R/Chang7-2 population; POP-BYB: B73/By804 population; LOD: Logarithm of odds; MAS: Marker-assisted selection; BLUP: Best linear unbiased prediction.

\section{Competing interests}

The authors declare that they have no competing interests.

\section{Authors' contributions}

LK carried out the experiments, analyzed data and wrote the manuscript; YJ carried out the field experiments; $L$ designed the study and assisted in writing the manuscript; YX designed the study and wrote the manuscript. All authors read and approved the final manuscript.

\section{Acknowledgements}

This study was supported by the National High-Tech Research and Development Program of China (2012AA101104) and the National Natural Science Foundation of China (31171561).

\section{Author details}

${ }^{1}$ National Maize Improvement Center of China, Beijing Key Laboratory of Crop Genetic Improvement, China Agricultural University, Beijing 100193, China. ${ }^{2}$ National Key Laboratory of Crop Genetic Improvement, Huazhong Agricultural University, Wuhan 430070, China.

Received: 24 February 2014 Accepted: 29 May 2014

Published: 3 June 2014

\section{References}

1. Flint-Garcia SA, Darrah LL, McMullen MD, Hibbard BE: Phenotypic versus markerassisted selection for stalk strength and second-generation European corn borer resistance in maize. Theor Appl Genet 2003, 107:1331-1336.

2. Flint-Garcia SA, Jampatong C, Darrah LL, McMullen MD: Quantitative trait locus analysis of stalk strength in four maize populations. Crop Sci 2003, 43:13-22.

3. Tuberosa R, Salvi S: QTL for agronomic traits in maize production. In Handbook of Maize: Its Biology. Edited by Bennetzen JL, Hake SC. New York: Springer; 2009:501-541.

4. Berzonsky W, Hawk J, Pizzolato T: Anatomical characteristics of three inbred lines and two maize synthetics recurrently selected for high and low stalk crushing strength. Crop Sci 1986, 26:482-488.

5. Martin M, Russell W: Correlated responses of yield and other agronomic traits to recurrent selection for stalk quality in a maize synthetic. Crop Sci 1984, 24:746-750.

6. Sibale E, Darrah L, Zuber M, Sleper D: Correlated responses to selection for stalk crushing strength in two maize populations. Maydica 1992, 37:115-122.

7. Thompson DL: Stalk strength of corn as measured by crushing strength and rind thickness. Crop Sci 1963, 3:323-329.

8. Twumasi-Afriyie S, Hunter R: Evaluation of quantitative methods for determining stalk quality in short-season corn genotypes. Can J Plant Sci 1982, 62:55-60.

9. Zuber $M$, Grogan $C:$ A new technique for measuring stalk strength in corn. Crop Sci 1961, 1:378-380.

10. Thompson D: Recurrent selection for lodging susceptibility and resistance in corn. Crop Sci 1972, 12:631-634.

11. Chesang-Chumo JJ: Direct and correlated responses to divergent selection for rind penetrometer resistance in MoSCSS maize synthetic In PhD thesis. University of Missouri-Columbia; 1993.

12. Dudley J: Selection for rind puncture resistance in two maize populations. Crop Sci 1994, 34:1458-1460.

13. Jampatong S, Darrah L, Krause G, Barry B: Effect of one-and two-eared selection on stalk strength and other characters in maize. Crop Sci 2000 40:605-611.

14. Kang MS, Din AK, Zhang Y, Magari R: Combining ability for rind puncture resistance in maize. Crop Sci 1999, 39:368-371.

15. McDevitt CM: Responses to three cycles of selection with a clamp for plant standability in two maize populations. In PhD thesis. University of Missouri-Columbia; 1999.

16. Sibale E, Darrah L, Zuber M: Comparison of two rind penetrometers for measurement of stalk strength in maize. Maydica 1992, 37:111-114.

17. Hu H, Meng $Y$, Wang $H$, Liu H, Chen S: Identifying quantitative trait loci and determining closely related stalk traits for rind penetrometer 
resistance in a high-oil maize population. Theor App/ Genet 2012, 124:1439-1447.

18. Peiffer JA, Flint-Garcia SA, De Leon N, McMullen MD, Kaeppler SM, Buckler ES: The genetic architecture of maize stalk strength. PLoS One 2013, 8:e67066.

19. Abedon BG, Darrah LL, Tracy WF: Developmental changes associated with divergent selection for rind penetrometer resistance in the MoSCSSS maize synthetic. Crop Sci 1999, 39:108-114.

20. Albrecht B, Dudley J: Divergent selection for stalk quality and grain yield in an adapted $\times$ exotic maize population cross. Crop Sci 1987, 27:487-494

21. Schnable PS, Ware D, Fulton RS, Stein JC, Wei F, Pasternak S, Liang C, Zhang J, Fulton L, Graves TA: The B73 maize genome: complexity, diversity, and dynamics. Science 2009, 326:1112-1115.

22. Chia J-M, Song C, Bradbury PJ, Costich D, de Leon N, Doebley J, Elshire RJ, Gaut B, Geller L, Glaubitz JC: Maize HapMap2 identifies extant variation from a genome in flux. Nat Genet 2012, 44:803-807.

23. Gore MA, Chia J-M, Elshire RJ, Sun Q, Ersoz ES, Hurwitz BL, Peiffer JA, McMullen MD, Grills GS, Ross-lbarra J: A first-generation haplotype map of maize. Science 2009, 326:1115-1117.

24. Rafalski A: Applications of single nucleotide polymorphisms in crop genetics. Curr Opin Plant Biol 2002, 5:94-100.

25. Buckler ES, Holland JB, Bradbury PJ, Acharya CB, Brown PJ, Browne C, Ersoz E, Flint-Garcia S, Garcia A, Glaubitz JC: The genetic architecture of maize flowering time. Science 2009, 325:714-718.

26. Poland JA, Bradbury PJ, Buckler ES, Nelson RJ: Genome-wide nested association mapping of quantitative resistance to northern leaf blight in maize. Proc Natl Acad Sci U S A 2011, 108:6893-6898.

27. Tian F, Bradbury PJ, Brown PJ, Hung H, Sun Q, Flint-Garcia S, Rocheford TR, McMullen MD, Holland JB, Buckler ES: Genome-wide association study of leaf architecture in the maize nested association mapping population. Nat Genet 2011, 43:159-162.

28. Cook JP, McMullen MD, Holland JB, Tian F, Bradbury P, Ross-lbarra J, Buckler ES, Flint-Garcia SA: Genetic architecture of maize kernel composition in the nested association mapping and inbred association panels. Plant Physiol 2012, 158:824-834

29. Li H, Peng Z, Yang X, Wang W, Fu J, Wang J, Han Y, Chai Y, Guo T, Yang N: Genome-wide association study dissects the genetic architecture of oil biosynthesis in maize kernels. Nat Genet 2013, 45:43-50.

30. Li Q, Yang X, Xu S, Cai Y, Zhang D, Han Y, Li L, Zhang Z, Gao S, Li J: Genome-wide association studies identified three independent polymorphisms associated with a-tocopherol content in maize kernels. PLoS One 2012, 7:e36807.

31. Pan Q, Ali F, Yang X, Li J, Yan J: Exploring the genetic characteristics of two recombinant inbred line populations via high-density SNP markers in maize. PLoS One 2012, 7:e52777.

32. Shutu X, Dalong Z, Ye C, Yi Z, Shah T, Ali F, Qing L, Zhigang L, Weidong W, Jiansheng $L$ : Dissecting tocopherols content in maize (Zea mays $L$.), using two segregating populations and high-density single nucleotide polymorphism markers. BMC Plant Biol 2012, 12:201

33. Jones $E$, Sullivan $H$, Bhattramakki D, Smith J: A comparison of simple sequence repeat and single nucleotide polymorphism marker technologies for the genotypic analysis of maize (Zea mays L.). Theor Appl Genet 2007, 115:361-371.

34. Yan J, Yang X, Shah T, Sánchez-Villeda H, Li J, Warburton M, Zhou Y, Crouch $\mathrm{JH}, \mathrm{Xu}$ Y: High-throughput SNP genotyping with the GoldenGate assay in maize. Mol Breed 2010, 25:441-451.

35. Yang X, Xu Y, Shah T, Li H, Han Z, Li J, Yan J: Comparison of SSRs and SNPs in assessment of genetic relatedness in maize. Genetica 2011, 139:1045-1054.

36. Yan J, Shah T, Warburton ML, Buckler ES, McMullen MD, Crouch J: Genetic characterization and linkage disequilibrium estimation of a global maize collection using SNP markers. PLoS One 2009, 4:e8451.

37. Ganal MW, Durstewitz G, Polley A, Berard A, Buckler ES, Charcosset A, Clarke JD, Graner E-M, Hansen M, Joets J: A large maize (Zea mays L.) SNP genotyping array: development and germplasm genotyping, and genetic mapping to compare with the B73 reference genome. PLoS One 2011, 6:e28334.

38. MaizeSequence. http://ensembl.gramene.org/Zea_mays/Info/Index.

39. Cobb JN, DeClerck G, Greenberg A, Clark R, McCouch S: Next-generation phenotyping: requirements and strategies for enhancing our understanding of genotype-phenotype relationships and its relevance to crop improvement. Theor Appl Genet 2013, 126:867-887.
40. Colbert T, Darrah L, Zuber M: Effect of recurrent selection for stalk crushing strength on agronomic characteristics and soluble stalk solids in maize. Crop Sci 1984, 24:473-478.

41. Ling G, Jianjun $H$, Rui S, Zaisong D, Zhiqiang D, Ming Z: Variation characteristic of stalk penetration strength of maize with different density-tolerance varieties. Trans Chin Soc Agr Eng 2010, 11:156-162.

42. Martin SA, Darrah LL, Hibbard BE: Divergent selection for rind penetrometer resistance and its effects on European corn borer damage and stalk traits in corn. Crop Sci 2004, 44:711-717.

43. Barrière $Y$, Méchin $V$, Lefevre B, Maltese S: QTLs for agronomic and cell wall traits in a maize RIL progeny derived from a cross between an old Minnesota13 line and a modern lodent line. Theor Appl Genet 2012, 125:531-549.

44. Krakowsky M, Lee M, Coors J: Quantitative trait loci for cell-wall components in recombinant inbred lines of maize (Zea mays L.) I: stalk tissue. Theor Appl Genet 2005, 111:337-346

45. Roussel V, Gibelin C, Fontaine A, Barriere Y: Genetic analysis in recombinant inbred lines of early dent forage maize. II. QTL mapping for cell wall constituents and cell wall digestibility from per se value and top cross experiments. Maydica 2002, 47:9-20.

46. Li L, Li H, Li Q, Yang $X$, Zheng D, Warburton $M$, Chai $Y$, Zhang $P$, Guo $Y$, Yan J: An 11-bp Insertion in Zea mays fatb reduces the palmitic acid content of fatty acids in maize grain. PLoS One 2011, 6:e24699.

47. Zhao Q, Nakashima J, Chen F, Yin Y, Fu C, Yun J, Shao H, Wang X, Wang $Z-Y$, Dixon RA: LACCASE is necessary and nonredundant with PEROXIDASE for lignin polymerization during vascular development in Arabidopsis. Plant Cell 2013, 25:3976-3987.

48. Berthet $S$, Demont-Caulet $N$, Pollet B, Bidzinski P, Cézard L, Le Bris P, Borrega $\mathrm{N}$, Hervé J, Blondet E, Balzergue S: Disruption of LACCASE4 and 17 results in tissue-specific alterations to lignification of Arabidopsis thaliana stems. Plant Cell 2011, 23:1124-1137.

49. Suzuki $K$, Suzuki $Y$, Kitamura S: Cloning and expression of a UDPglucuronic acid decarboxylase gene in rice. J Exp Bot 2003, 54:1997-1999.

50. Lee C, Teng Q, Zhong R, Ye Z-H: Arabidopsis GUX proteins are glucuronyltransferases responsible for the addition of glucuronic acid side chains onto xylan. Plant Cell Physiol 2012, 53:1204-1216.

51. Hongo S, Sato K, Yokoyama R, Nishitani K: Demethylesterification of the primary wall by PECTIN METHYLESTERASE35 provides mechanical support to the Arabidopsis stem. Plant Cell 2012, 24:2624-2634.

52. Alsirt A: Evaluation of frequency distribution changes resulting from bi-directional selection for rind penetrometer resistance in maize. In PhD thesis. University of Missouri-Columbia; 1993.

53. Masole $\mathrm{H}$ : Evaluation of high and low divergent rind penetrometer resistance selection at three plant densities in maize. In PhD thesis. University of Missouri-Columbia; 1993.

54. Lande R, Thompson R: Efficiency of marker-assisted selection in the improvement of quantitative traits. Genetics 1990, 124:743-756.

55. Mohan M, Nair S, Bhagwat A, Krishna T, Yano M, Bhatia C, Sasaki T: Genome mapping, molecular markers and marker-assisted selection in crop plants. Mol Breed 1997, 3:87-103.

56. Collard BC, Mackill DJ: Marker-assisted selection: an approach for precision plant breeding in the twenty-first century. Philos Trans $R$ Soc Lond Ser B Biol Sci 2008, 363:557-572.

57. Huang N, Angeles E, Domingo J, Magpantay G, Singh S, Zhang G, Kumaravadivel N, Bennett J, Khush G: Pyramiding of bacterial blight resistance genes in rice: marker-assisted selection using RFLP and PCR. Theor Appl Genet 1997, 95:313-320.

58. Zhao X, Tan G, Xing Y, Wei L, Chao Q, Zuo W, Lübberstedt T, Xu M: Marker-assisted introgression of $q H S R 1$ to improve maize resistance to head smut. Mol Breed 2012, 30:1077-1088.

59. Holland JB, Nyquist WE, Cervantes-Martínez CT: Estimating and interpreting heritability for plant breeding: An update. Plant Breed Rev 2003, 22:9-112

60. Knapp S, Stroup W, Ross W: Exact confidence intervals for heritability on a progeny mean basis. Crop Sci 1985, 25:192-194.

61. Murray M, Thompson WF: Rapid isolation of high molecular weight plant DNA. Nucleic Acids Res 1980, 8:4321-4326.

62. Fan JB, Gunderson KL, Bibikova M, Yeakley JM, Chen J, Wickham Garcia E, Lebruska LL, Laurent M, Shen R, Barker D: [3] Illumina Universal Bead Arrays. Methods Enzymol 2006, 410:57-73.

63. Purcell S, Neale B, Todd-Brown K, Thomas L, Ferreira MA, Bender D, Maller J, Sklar P, De Bakker PI, Daly MJ: PLINK: a tool set for whole-genome 
association and population-based linkage analyses. Am J Hum Genet 2007, 81:559-575.

64. Van Ooijen J: JoinMap 4. In Software for the Calculation of Genetic Linkage Maps in Experimental Populations Kyazma BV, Wageningen, Netherlands; 2006.

65. Basten CJ, Weir BS, Zeng Z-B: QTL cartographer: a reference manual and tutorial for QTL mapping. In Department of Statistics. Raleigh, NC: North Carolina State University; 1997.

66. Lander ES, Botstein D: Mapping mendelian factors underlying quantitative traits using RFLP linkage maps. Genetics 1989, 121:185-199.

67. Kao C-H, Zeng Z-B, Teasdale RD: Multiple interval mapping for quantitative trait loci. Genetics 1999, 152:1203-1216.

68. Quevillon E, Silventoinen V, Pillai S, Harte N, Mulder N, Apweiler R, Lopez R: InterProScan: protein domains identifier. Nucleic Acids Res 2005, 33:W116-W120.

doi:10.1186/1471-2229-14-152

Cite this article as: Li et al.: Genetic architecture of rind penetrometer resistance in two maize recombinant inbred line populations. BMC Plant Biology 2014 14:152.

\section{Submit your next manuscript to BioMed Central and take full advantage of:}

- Convenient online submission

- Thorough peer review

- No space constraints or color figure charges

- Immediate publication on acceptance

- Inclusion in PubMed, CAS, Scopus and Google Scholar

- Research which is freely available for redistribution 